\title{
Current Source Reconstruction with Independent Component Analysis in Sensor Array Measurement of Multi-Conductor Current
}

Ayambire $\mathbf{P N}{ }^{*}$ and Huang $\mathbf{Q}$

Department of Electronic Science, University of Electronic Science and Technology of China, Qingshuihe Campus, China

\begin{abstract}
This paper implemented a current reconstruction system based on independent component analysis and magnetic field sensors. In this paper both the normal approximation and interfered approximation of magnetic flux density generated by current carrying conductors and sensed by four biaxial TMR sensors are presented and discussed. The current in each conductor with and without interference was calculated based on the comprehensive independent component analysis algorithm. The error for both the simulated and numerical calculations results was within acceptable levels for protective devices proving the feasibility of our reconstruction system.
\end{abstract}

Keywords: Current reconstruction; Interference fields; Sensor array; Tunnel magnetoresistive sensor

\section{Introduction}

Multi-conductor current reconstruction is one of the major challenges in modern power grids which could lead to major instability in the grid. Power transmission lines have made an important contribution to long-distance power transmission and distribution in power grids [1]. However, current reconstruction in power transmission lines is very challenging as a result of interference created by each conductor within the vicinity. The use of magnetic sensors for current reconstruction is widely adopted by many researchers and has offered many solutions based on the Hall Effect, GMR and AMR sensors [2]. Manara A et al. present examples of magnetic sensor circular arrays. The sensors are mounted in a circular form around the conductor whose current is under estimation. In order to obtain meaningful interference reduction, an approximation of Ampere's circuital law is performed making the sum of the sensor output signals [3].

Apart from the above mentioned current estimation methods; there is also a lot of research work about methods of current estimation. In an apparatus for estimating current using magnetic sensors which are symmetrically mounted on a conductor and the ambient interference were eliminated by computing the average sum of the sensors outputs [4]. Manara A and Weiss R proposed a method for applying coreless Hall effect sensors to estimate current in large cables, with focus on external interference $[3,5]$. In many of these methods, the magnetic sensors are directly fixed to the cable. To establish the effect of conductor shape, diameter, insulation thickness and installation methods on the measured magnetic field strength, then sensor calibration will have to be done. Chan JYC presents a more flexible method for estimating current in circular conductors by a coreless sensor which makes use of a micro-controller unit for the automatic sensing calibration [6]. Nevertheless, the above works all deal with single conductor current estimation. It becomes a more challenging issue when it comes to the estimation of multi-conductor current. According to Chan JYC estimating the current of a single conductor can be easily analyzed by implementing sensor array [6]. Even though magnetic sensor arrays can be used for multi-conductor current estimation, it is however very difficult to differentiate the current estimation of each conductor under measurement. In 7 , how to decouple cross-coupled fields in current sensor arrays was done [7]. This methodology, however, requires the prior knowledge of the geometrical properties of the conductor.

Douglas P and McNutt proposed another method that utilized a computational method to determine the positions of the conductors by solving an inverse problem. However, the accuracy of current measurement was not examined since there are difficulties in obtaining the technical details of this method $[8,9]$.

In offering solution to the drawbacks in the previous studies, a novel method based on the use of independent component analysis algorithm and tunnel magnetoresistive (TMR) sensor.

\section{Formulation of Current Estimation Problem}

Consider a circular array made up of biaxial tunnel magnetoresistive (TMR) sensors equally distributed around a linear conductor of which the current flowing in the conductor is to be estimated as shown in Figure 1, the center of the circle coincides with the conductor axis. From Ampere's law, the magnetic flux density, B(t) detected by the two-axis sensors placed around the current carrying conductor is approximated as;

$$
\left\{\begin{array}{c}
B_{x}(t)=\frac{\mu_{o} I(t)}{2 \pi R} \\
B_{y}(t)=0
\end{array}\right.
$$

Where $\mathrm{B}_{\mathrm{x}}$ and $\mathrm{B}_{\mathrm{y}}$ are the magnetic flux density detected by the sensors $x$-sensitive axis and $y$-sensitive axis respectively, $\mu$ is the permeability of free space, is the magnitude of the current and is the radius of the array [10].

From Ampere circuital law, (1) is an approximation of which the estimation accuracy of the array would be affected by interference fields that may originate from nearby conductors. It is as a result of this that there is the need to establish an accurate way of reconstruction signals in a multi-conductor with negligible interference.

*Corresponding author: Ayambire PN, Department of Electronic Science University of Electronic Science and Technology of China, Qingshuihe Campus: No. 2006, Xiyuan Ave., West High-tech Zone, Chengdu, Sichuan, China, Tel: +8615528125089; E-mail: nayambire@ymail.com

Received July 05, 2018; Accepted July 19, 2018; Published July 26, 2018

Citation: Ayambire PN, Huang Q (2018) Current Source Reconstruction with Independent Component Analysis in Sensor Array Measurement of Multi-Conductor Current. J Electr Electron Syst 7: 264. doi: 10.4172/2332-0796.1000264

Copyright: (c) 2018 Ayambire PN, et al. This is an open-access article distributed under the terms of the Creative Commons Attribution License, which permits unrestricted use, distribution, and reproduction in any medium, provided the original author and source are credited. 
Citation: Ayambire PN, Huang Q (2018) Current Source Reconstruction with Independent Component Analysis in Sensor Array Measurement of Multi-Conductor Current. J Electr Electron Syst 7: 264. doi: 10.4172/2332-0796.1000264

Page 2 of 5

Figure 2 present a multi-conductor situation where there is interference field originating from conductor $\mathrm{I}_{2}$. The presence of conductor $\mathrm{I}_{2}$ reduces the reconstruction accuracy of (1) since fields generated by it are picked up by the sensors around conductor $\mathrm{I}_{1}$. However, based on the linearity of the Biot - Savart's law, the resultant interference error created by multi-current is the superposition of the interference errors due to each current. Hence, it is more convenient to consider the interference error radiated by a long infinite conductor parallel to the current under measurement. Mathematically, these interferences can be expressed as;

$$
\begin{aligned}
& B_{\text {noisey } 1}(t)=\frac{\mu_{o} I_{2}(t)}{2 \pi(D+R)} \cos \alpha \\
& B_{\text {noisey } 2}(t)=\frac{\mu_{o} I_{2}(t)}{2 \pi(D+R)} \sin \alpha
\end{aligned}
$$

From the illustration in Figure 2, the magnetic field detected by each sensor when there is current flowing in both conductors, $I_{1}$ and $\mathrm{I}_{2}$ is expressed as;

$$
\left\{\begin{array}{l}
\left\{B_{x}(t)=B_{x}(t)+B_{\text {noise } 1}(t)\right. \\
\left\{B_{y}(t)=B_{y}(t)+B_{\text {noise } 2}(t)\right.
\end{array}\right.
$$

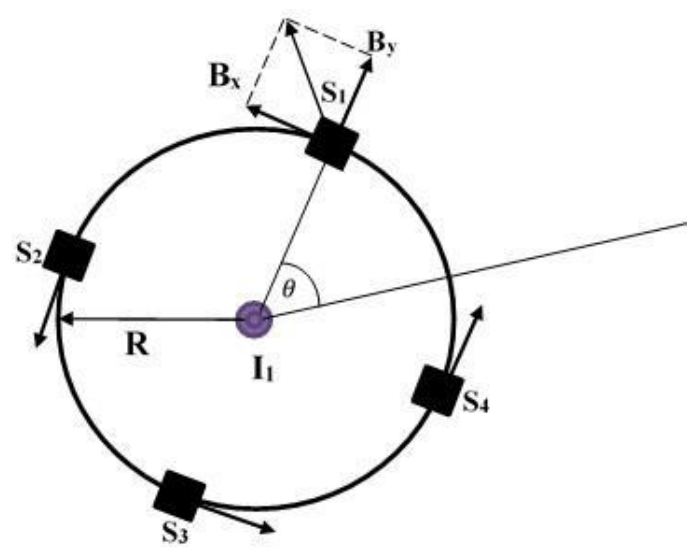

Figure 1: Modeling of a single conductor with sensor array system.

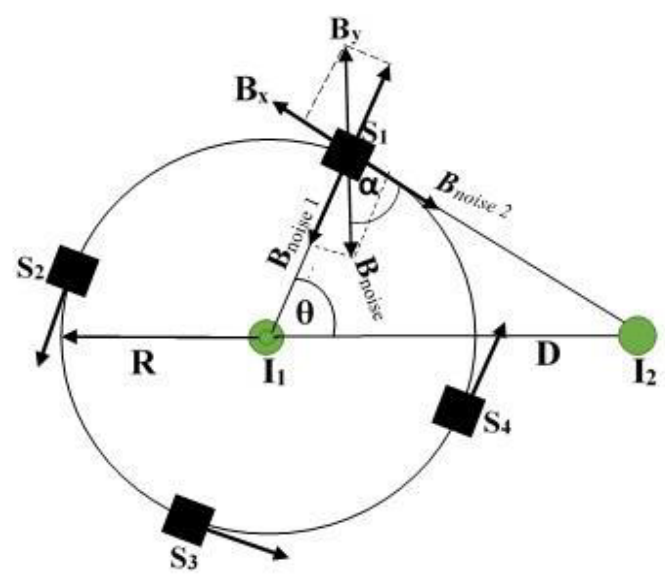

Figure 2: Modeling of multi-conductor with sensor array system.
In Figure 2, all the sensors (from 1 to 4 ) are reacting only to the magnetic component parallel to their sensitive axes. In addition, the sensitivities $(S)$ is considered to be identical for all the sensors. The sensors are assumed to be placed in an ideal symmetry and equidistance around the conductor $\left(\mathbf{I}_{1}\right)$, the current under estimation so that their centers are on one concentric circle. One sensitive axis of each sensor is tangential to the circle at this point. This implies that magnetic field will only be detected by the sensor on the $x$ - sensitive axis and, on the $y$-axis detection $\mathbf{B}_{\mathbf{y}}(\mathbf{t})=\mathbf{0}$. Therefore, the magnetic field detected by the sensors when there is current flowing through both conductors, $\mathbf{I}_{1}$ and $I_{2}$ can be expressed as;

$$
\left\{\begin{array}{c}
B_{x}(t)=B_{x}(t)+B_{\text {noise }}(t) \\
B_{x}(t)=\frac{\mu_{o} I(t)}{2 \pi R}+\frac{\mu_{o} I_{2}(t)}{2 \pi(D+R)} \cos \alpha
\end{array}\right.
$$

Assuming the current under estimation to be unitary and using ANSYS Maxwell simulations, we have plotted the magnetic fields generated in two scenarios; Figure 3a shows the magnetic fields picked up by the sensor array without strong interference sources and Figure $3 \mathrm{~b}$ shows the magnetic fields picked up by the sensor array with interference source from a nearby current carrying conductor. As a result of the interference created by the conductor $\mathrm{I}_{2}$, the approximation accuracy of (1) is affected as shown in Figures 4-6.

\section{Background to Source Separation with Sensor Array}

Source separation is a mathematical technique used for extracting hidden parameters that underlie in sets of random variables or signals. This technique is based on the assumption that signals from different sources are statistically independent and statistically independent sources can be extracted from mixed signals. Therefore, the condition of source statistical independence must be fulfilled to the success of this technique.

Source separation defines a model for the observed data that requires a set of data samples in order to establish the necessary statistics. The assumption that the data variables are a linear combination of unknown variables, the unknown variables are assumed to be non-Gaussian and

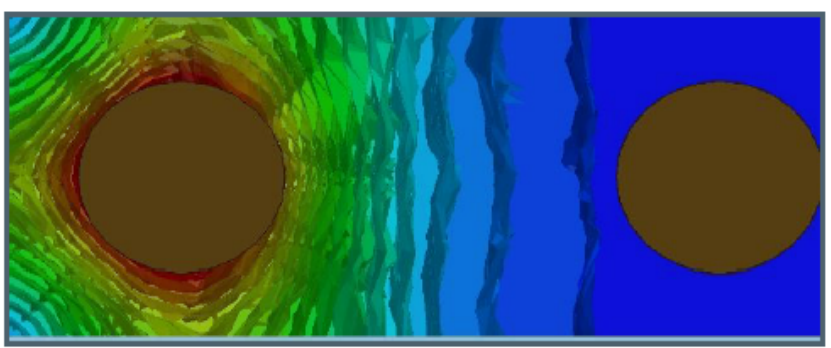

Figure 3a: Magnetic fields of the sensor array.

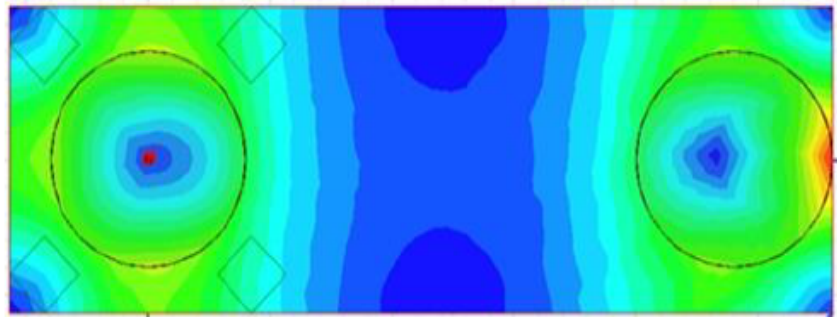

Figure 3b: Magnetic fields of the sensor array with the Interference source $\mathrm{I}_{2}$. 
Citation: Ayambire PN, Huang Q (2018) Current Source Reconstruction with Independent Component Analysis in Sensor Array Measurement of Multi-Conductor Current. J Electr Electron Syst 7: 264. doi: 10.4172/2332-0796.1000264

Page 3 of 5

independent. The objective then is to find a transformation in which the components are as statistical independent from each other as possible.

The main objective of the source separation and reconstruction algorithm is to recover the unobserved original signals from multiple signal mixtures. Generally, the observed signals are the output of a set of sensors, each sensor receiving different original mixed signals.

An example of the deployment of the source separation and construction technique is when there is a mixture of simultaneous signals that have been picked up by a magnetic sensor array; it is desirable to have the original signals isolated. One way of doing this is through an algorithm implemented in ICA. The algorithm accumulates statics of the incoming signals and then analyzes them, trying to isolate non-Gaussian behavior and independent characteristics that the signal can present. The ICA algorithm can distinguish as many sources as independent variables are generated by the linear combination of the sources.

In this paper, a source reconstruction system is implemented based on the independent component analysis algorithm.

During the pre-processing stage, the covariance matrix of the input signals is linearly transformed to be equal to an identity matrix as given below;

$$
\operatorname{Cov}(V, M)=1
$$

Where $V$ is the new variable with the whitened data, $M$ the variables containing the data and is the identity matrix. After the preprocessing, the data enters the comprehensive based independent component analysis algorithm. At this stage there are several methods of implementing the algorithm, these methods mostly involve the maximization of non-Gaussianity variables fed into the algorithm.

\section{Sensor Output Voltage as Mixture for Source Separation}

Using the illustration in Figure 2, the following assumptions are made to enable the successful implementation of the algorithm on the problem. From Figure 2, $I_{1}$ and $I_{2}$ are represented by $S_{1}$ and $S_{n}$ respectively, $v_{\mathrm{i}}(\mathrm{t})$ to $v_{m}(\mathrm{t})$ represents the outputs of sensors 1 to $m$, and $y_{1}(\mathrm{t})$ and $y_{\mathrm{n}}(\mathrm{t})$ represents the estimated reconstruction of $S_{1}$ and $\mathrm{S}_{\mathrm{n}}$ Figure 7.

The output signal delivered by a magnetic sensor is an unknown superimposition of the inputs of the various sources. The output signals $(t), i=1, \ldots$, of a sensor array can be considered as linear instantaneous mixtures of the unknown source signals $s_{i}(t), i=1, \ldots, n$.

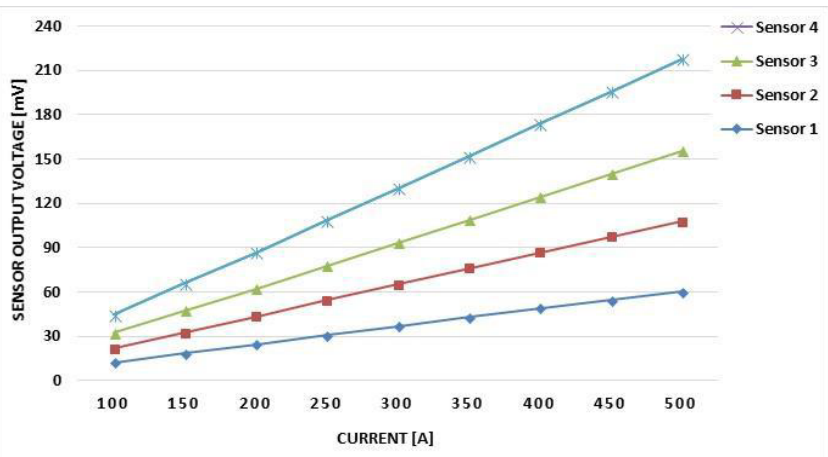

Figure 4: Output characteristic of the individual sensors of the circular sensor array without strong interference sources.

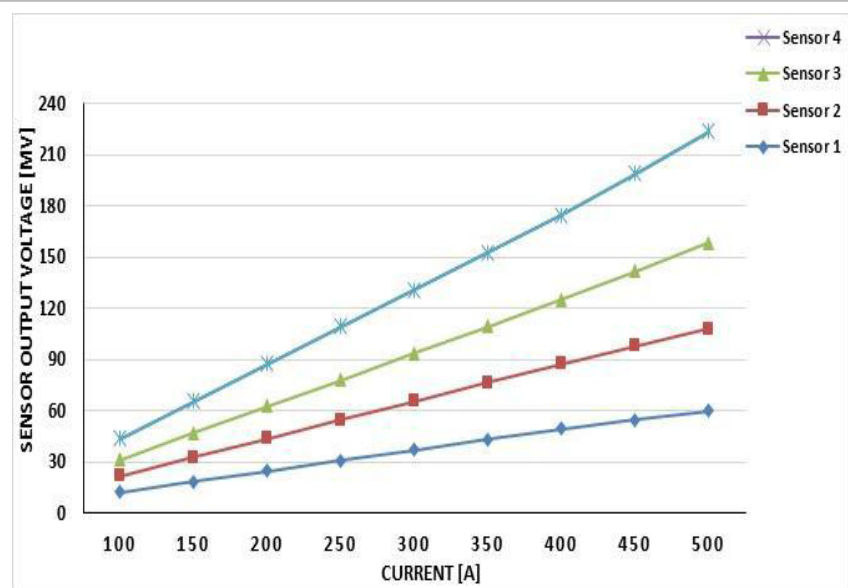

Figure 5: Output characteristic of the individual sensors of the circular senso array with the interference source 12 at a distance of $120 \mathrm{~mm}$.

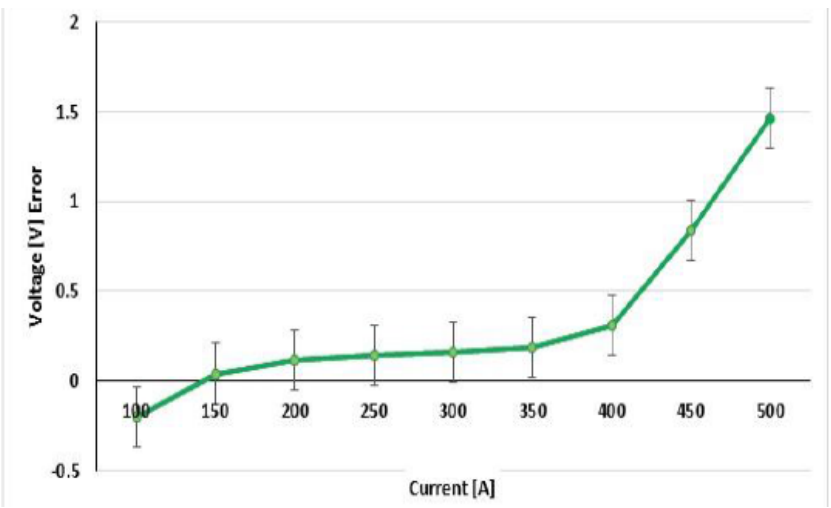

Figure 6: Approximation error due to the presence of 12 , where 12 is at a distance of $120 \mathrm{~mm}$ from the center of the array.

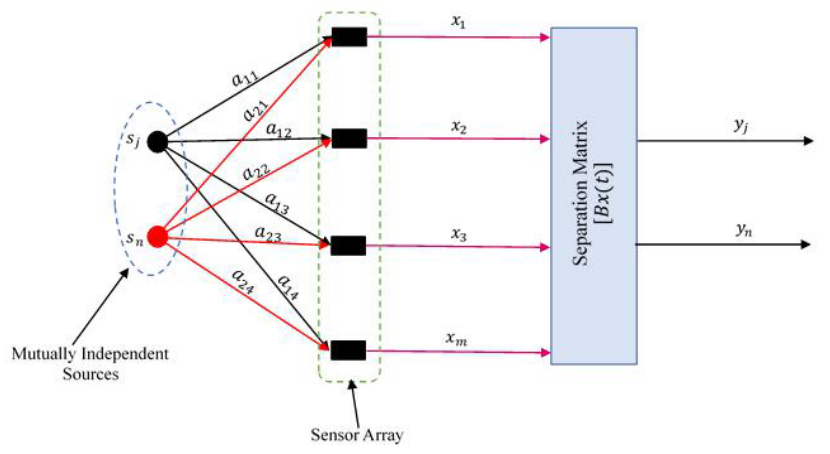

Figure 7: Independent component analysis model.

$$
\begin{aligned}
& v_{i}(t)=\sum_{j=1}^{m} a_{i j} s(t) \\
& V(t)=A s(\mathrm{t})
\end{aligned}
$$

Where $s(t)$ and $A$ are unknown

A source separation algorithm consists in estimating a de-mixing or separation matrix, $B(t)$ whose output should be an estimation of the original source signals; 


$$
\left\{\begin{array}{c}
y(t)=B(t) v(t) \\
y(t)=B(t) A s(t) \\
y(t)=\hat{s}(t)
\end{array}\right.
$$

The observed mixtures $(t), i=1, . . m$ are not modified by scale changes and permutation;

$$
v_{i}(t)=\sum_{j=1}^{m} \frac{a_{i} \sigma(j)}{c \sigma(j)}[z \varphi(j) s \varphi(j)(t)]
$$

Where $z \phi(j)$ is a constant, $\phi($.$) is a permutation of 1, \ldots, n$ The source cannot be exactly estimated but only up to a permutation and a scale factor;

$$
\mathrm{y}(t)=P \operatorname{Ds}(t)
$$

Where $D$ is an $n \times n$ diagonal matrix with non - zero entries.

$P$ is an $n x n$ permutation matrix.

The magnetic sensor output is a complex function of input from various magnetic field sources;

$$
V_{\text {output }}=f\left(B_{1}, \ldots, B_{k}\right)
$$

The voltage of a magnetic sensor is given by;

$$
V_{\text {output }}=S B \sin \alpha
$$

Where $S$ is the sensor sensitivity which is equal for all the sensors, is the magnetic field density and is the angle of incidence of the magnetic field.

For a given sensor, differentiating at first order;

$$
\left\{\begin{aligned}
d V_{\text {output }} & =S \frac{d B_{1} \sin \alpha_{1}}{d V_{\text {output }}} \mid B_{i, i \neq 1}^{*} V_{\text {output }} B_{1} \\
& +, \ldots .,+S \frac{d B_{1} \sin \alpha_{1}}{d V_{\text {output }}} \mid B_{i, i \neq n}^{*} V_{\text {output }} B_{m}
\end{aligned}\right.
$$

Where $V_{\text {output }} B_{1}, \ldots, V_{\text {output }} B_{m}$ are the equivalent induced sensor voltage by $n$ independent variable magnetic sources, $B 1, \ldots, B m$ weighted at the sensor array output by the unknown entries of the mixing matrix $A$.

\section{Tmr Sensor Array Processing}

From equation (13), the output of the sensor array is modeled as;

$$
V_{\text {output }}(\mathrm{t})=A V_{\text {output }} B(t)+n(t)
$$

Where $V_{\text {output }}(t)=\left[V_{\text {output } 1}(t), \ldots, V_{\text {outputm }}(t)\right]^{\mathrm{T}}$ is the vector of the observed voltages at the sensor array output, $V_{\text {output }} B(t)=\left[V_{\text {output }} B_{1}(t)\right.$, $\left.\ldots, V_{\text {output }} B_{n}(t)\right]^{\mathrm{T}}$ is the vector of the induced voltages by $n$ magnetic field sources and the vector $n(t)=\left[n_{1}(t), n_{2}(t), . ., n_{m}(t)\right]^{T}$ corresponds to the additive electronic noise assumed to be Gaussian or spatially white and non - correlated with $V_{\text {output }} B(t)$.

\section{Simulation Results}

In this paper, the capacities of the algorithm for multi-conductor current reconstruction are analysis by way of analytical and numerical simulations. The use of ICA algorithm can provide redundant information. When the system feeds more signals into the algorithm than real signals from the environment, the algorithm will provide a redundant solution.
To validate the algorithm for source reconstruction in a multiconductor current situation, a test was set up using the (15) where two independent magnetic field sources were detected by four tunnel magnetoresistive (TMR) sensors forming a mixture of two sinusoidal waves.

$$
\left\{\begin{array}{c}
S_{1}=B_{1} \sin [2 \pi f(t)] \\
S_{2}=B_{2} \sin \left[2 \pi f(t)+\frac{2}{3} \pi\right]
\end{array}\right.
$$

Where $B_{1}$ and $B_{2}$ are the magnetic flux density generated by the current of conductors $I_{1}$ and $I_{2}$ respectively, $f$ is the frequency and $S_{1}$ and $S_{2}$ are the sine wave created by current of conductor $I_{1}$ and $I_{2}$.

The individual sensor behavior was evaluated as a function of the magnetic flux density after which the average magnetic flux density of the sensors was calculated using ANSYS Maxwell Figures 8-13.

A numerical simulation was then done in MATLAB using four TMR sensors forming a sensor array and two magnetic field sources. Figure 11 shows the simulation of the mixed signals picked up by the four sensors taken into account additive noise. However, the adaptive algorithm performed as expected and eliminated two similar vectors.

The signal separation and reconstruction were performed numerically in MATLAB using the independent component analysis. The algorithm processes the sensors mixed signals that appeared on the sensor output. The Figure 11 shows the reconstructed signal which was performed using the independent component analysis algorithm. With the information obtained from the observed mixtures, the algorithm allows us to estimate the TMR output.

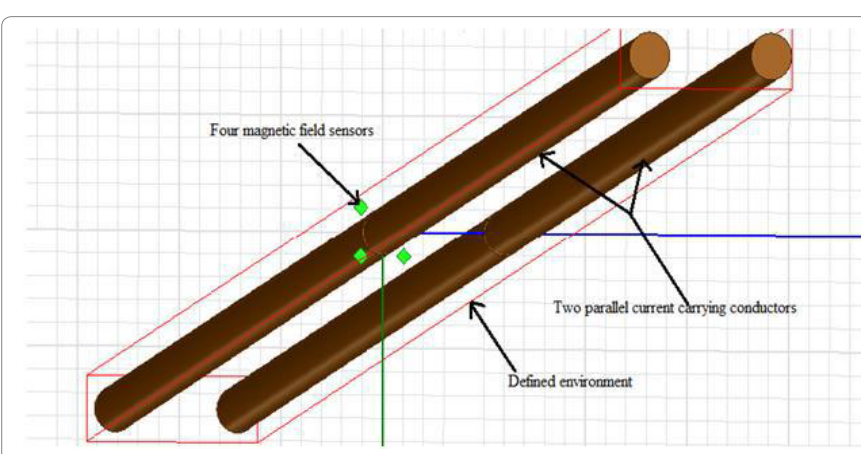

Figure 8: ANSYS Maxwell simulation of two parallel current carrying conductors and four magnetic sensors surrounding the conductor under measurement.

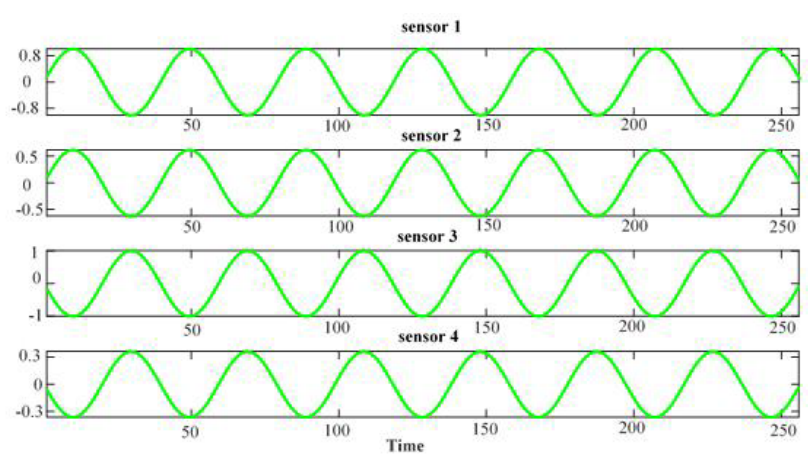

Figure 9: Mixed Source Signals, $V_{\text {output }}(m)(i=1, \ldots, 4)$, Sensor outputs. 


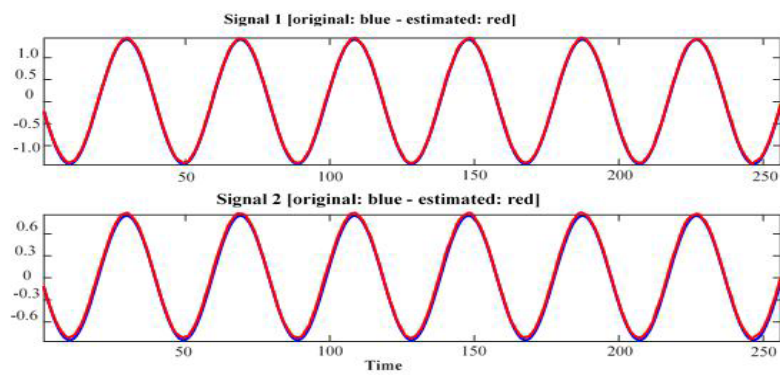

Figure 10: Reconstructed source signals $\left[\mathrm{Y}_{1}(\mathrm{t})\right.$ and $\left.\mathrm{Y}_{2}(\mathrm{t})\right]$

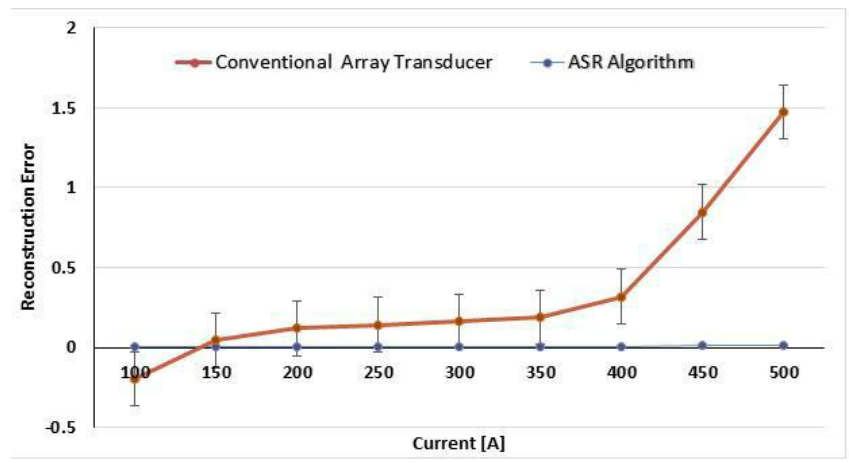

Figure 11: Reconstructed source signals error where $I_{2}$ is $120 \mathrm{~mm}$ from the center of the array.

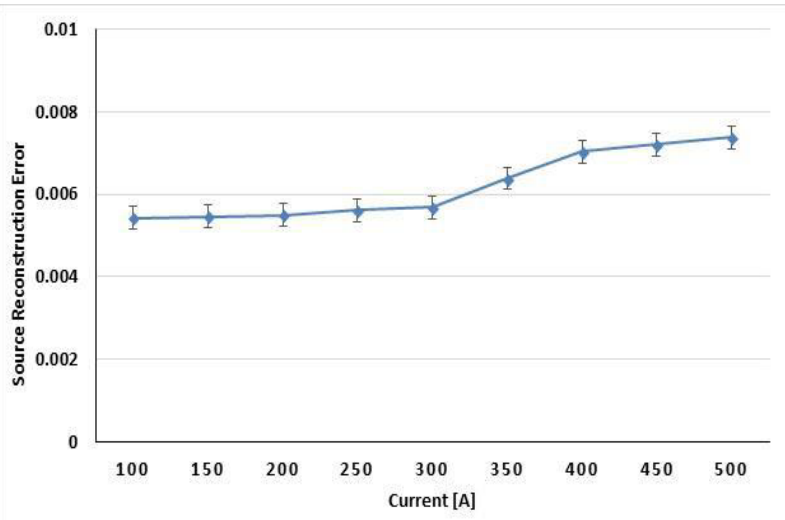

Figure 12: Estimation error of source signals at different angles $\alpha^{0}$. This figure shows the dependence on distance for two angles $0^{\circ}$ and $270^{\circ}$.

The algorithm results showed that the algorithm is very reliable for the system. The calibration of these coefficients is straight forward and uses a sinusoidal wave and the sensor electronic noise.

\section{Conclusion}

This study demonstrates the implementation of a signal separation and AC current estimation system in multi-conductor environment based on a neural independent component analysis a method for solving Blind Source Separation problem in a real-time situation. The currents are calculated by using a set of TMR magnetic sensor array measurement and an adaptive algorithm based on neural network independent component analysis and Pearson product-moment correlation coefficient. The proposed technique represents an improved

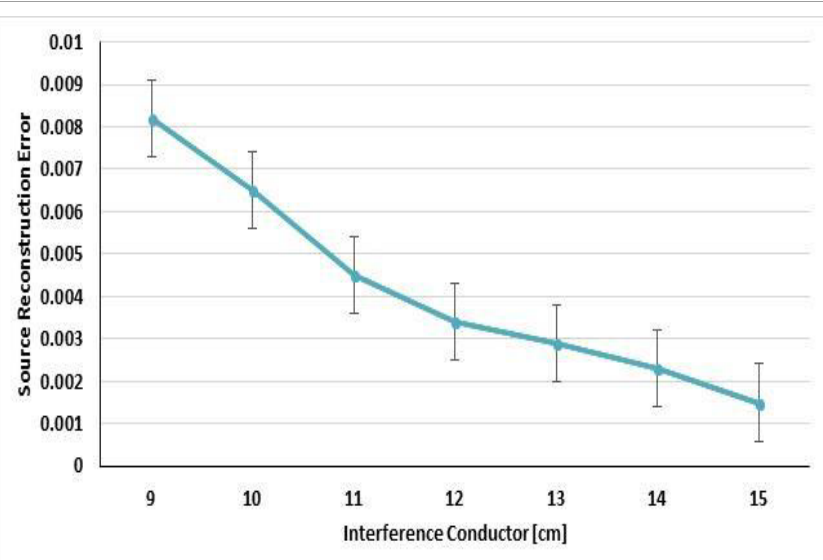

Figure 13: Source reconstruction error vs. Interference conductor distance ICA.

way to overcome the major interference issues posed by individual conductor current in multi-conductor current estimation systems.

Results from both ANSYS Maxwell and MATLAB involving the array data and performing source estimation were provided and studied. The results proved that it is possible to performed signal separation and current estimation with sensor array implemented in this adaptive algorithm.

The developed algorithm first carries out signal separation and the current estimation. We formulated the problem using analytical simulation based on ANSYS Maxwell and performed the separation and current estimation using numerical simulation based on MATLAB. After testing the algorithm on a number of situations, the error for signal separation and current estimation remains far less than $1 \%$ which is acceptable for protective devices.

\section{References}

1. Zhao G, Hu J, Zhao S, Wang Z, Wang SX, et al. (2016) Tunneling Magnetoresistive Sensors for High-Frequency Corona Discharge Location. IEEE Trans Magn 52: 1-1.

2. Sun X, Huang Q, Jiang LJ, Pong PWT (2014) Overhead High-Voltage Transmission-Line Current Monitoring by Magnetoresistive Sensors and Current Source Reconstruction at Transmission Tower. IEEE Trans 50: 1-4.

3. Manara A, Di Rienzo L, Ottoboni R (2001) Low-Cost Current Transducer Based On Circular Array of Magnetic Sensors. Trends in Electrical Measurement and Instrumentation 1: 135-139.

4. Weiss R, Makuch R, Itzke A, Weigel R (2017) Crosstalk in Circular Arrays of Magnetic Sensors for Current Measurement. IEEE Trans Ind Electron 64: 4903-4909.

5. Chan JYC, Member S, Tse NCF, Lai LL (2013) A Coreless Electric Current Sensor With Circular Conductor Positioning Calibration. IEEE Trans Instrum Meas 62: 2922-2928.

6. Bazzocchi R, Di Rienzo L (2000) Interference rejection algorithm for current measurement using magnetic sensor arrays. Sens Actuators A Phys 85: 38-41.

7. Kojovic LA, Beresh R, Bishop MT, Javora R, Magruder B, et al. (2010) Practical Aspects of Rogowski Coil Applications to Relaying. IEEE PSRC Spec Rep 62 $1-72$.

8. McNutt DP (2000) Current Measurement. Copyright 2000 CRC Press LLC.

9. Dongwei L, Di Rienzo L (2011) Robustness Analysis of Magnetic Sensor Arrays for Current Sensing. 1st International Conference on Electric Power Equipment - Switching Technology, pp: 670-673.

10. Li D, Di Rienzo L, Wang J, Zhang Z (2012) Current measurement in the time domain based on the inversion of magnetic field data. Inverse Probl Sci Eng 20: 3-14. 